\title{
Dynamics and Modeling for Unification of Mechanical and Electrical Systems
}

\author{
Akio Nagamatsu \\ Mechanical Engineering Department \\ Hosei University \\ 3-7-2 Kajino-cho, Kogenei-shi, Tokyo 184-8584, \\ JAPAN \\ E-Mail: nagamatu@k.hosei.ac.jp
}

Keywords: Dynamics, Modeling, Functional Model, Mechanical and Electrical Systems

\begin{abstract}
An approach on modeling based on unified dynamics over mechanical and electrical systems is put forward for fully computer aided design and development of machine products. A concept of the functional model is proposed for embodying this approach. The proposed model is used mainly for simulating the functions and the efficiencies of the machine composed of mechanical and electrical parts. The functional models of some mechanical and electrical systems are illustrated.
\end{abstract}

\section{Introduction}

Many machines, for example hybrid engine in automobile, are activated using both of mechanical and electrical energies, so in designing them some approach on modeling is necessary for explaining these two types of energies collectively in one manner. The function and the efficiency of machine have to be planed, designed and optimized before its structure and dimension are determined in its development. So, some model with which function can be checked before shape and structure is given is indispensable

\author{
Shizuo Sumida \\ CATEC Inc. \\ 5-16-3 Asakusabashi, Taito-ku, Tokyo 111-0053, \\ JAPAN \\ E-Mail: $\quad$ sumida.shi@guest.mazda.co.jp
}

for this activity. It is important and difficult problem that we have no approaches for constructing model making this possible. All conventional approaches on modeling, for example the finite element method, are developed not for creation of function but for analysis of phenomenon, so the conventional approaches of modeling are little valid for functional development of machine.

A new approach on modeling is put forward by the author in order to solve this problem, and a basic concept of the functional model is proposed ${ }^{(1)-(7)}$. In this report first, the properties of the model necessary for functional development of machine are listed. Second, unified expression method of mechanical and electrical dynamics is introduced. Third, the basic concept of the functional model is introduced. Fourth, simple functional models of some mechanical and electric systems are illustrated.

\section{Property of the Model Necessary for Functional Development}

1) Different types of energies, for example mechanical and electrical energies, can be treated in unification in one model.

2) The model explains not the structure but the function of the object, and is assembled faithfully according to energy flow in it. 
3) All explanations of both inputs and outputs are standardized, and expansion of the model can be done by connection of parts.

4) The model is of a hierarchical composition. The model is abstract and shows the function of the total system at the upper level. It is concrete and relates the function with the structure at the lower level.

5) All operations can be simulated from the upper to the lower streams of development through plan, design, prototyping and testing.

6) Nonlinear characteristics of the object can be explained with the model correctly and easily.

7) Functions and phenomena in multiple physical fields such as mechanical and electric ones can be explained, connected and treated by an integrative manner.

8) Mathematical treatment can be performed automatically with the computer.

Conventional models have not the above properties, and are not valid for functional creation of machine.

\section{Dynamics Common in Mechanical and Electrical Systems}

In general, energy system is composed of two types of state values, namely quantitative and qualitative ones. The authors call the former as potential value and the latte as flow value. In mechanical systems the former is velocity and the latter is force, and in electrical systems the former is voltage and the latter is electric current. Multiplication of these two values becomes energy. The former is ruled by the low of continuity and the latter is ruled by the low of equilibrium. For example, velocity continuity and force equilibrium in mechanical system, and voltage continuity ( Kirchhoff's second low ) and electric current equilibrium ( Kirchhoff's first low ) in electrical system. Three kinds of characteristics compose dynamics of energy system. For example, mass, stiffness and viscosity in mechanical system, and capacitance, inductance and resistance in electrical systems. These facts make unified modeling of mechanical and electrical systems possible.

\section{Functional Model}

A new concept of the functional model is proposed by the author in order to solve the problems in conventional models, and to model mechanical and electrical systems in unification.

The authors' model is explained with a block diagram for explaining the function and the operation, and for visual understanding of energy flow in the object system. It accompanies mathematical model composed of a series of government equations for analysis and treatment.

Three kinds of physical values are used in the model, namely the state value, the characteris tic and the coefficient. The state values explain the state of the object, and are divided into the flow value, for example electric current and force, and the potential value, for example voltage and velocity. The product of the flow and the potential values becomes the energy as mentioned above.

The characteristics explain the properties of the object, and relate the flow and the potential values. The characteristics are divided into the storage characteristic, for example inductance, capacitance, stiffness and mass, and the loss characteristic, for example resistance and damping.

The coefficients relate between the flow values or between the potential values, for example gear ratio and motor constant.

In order to illustrate the functional composition of the object in visually understandable way, some kinds of symbols are adopted in the block diagram. Figure 1 shows the main symbols and their meanings. A pear of a flow value and a potential value becomes the input and output. If one of these two is input, the other is output. The product of input and output becomes energy. The functional model is made in principle by combining the unit functional elements some typical examples of which are shown in Fig. 2. 


\begin{tabular}{|c|c|}
\hline$\longrightarrow$ & Potential value \\
\hline$\rightarrow$ & Flow value \\
\hline$\rightarrow$ & Signal value \\
\hline - $C$ & $C=A+R$ \\
\hline$\Rightarrow$ & $C=A \times B$ \\
\hline $\begin{array}{l}\Rightarrow A \\
\Rightarrow A\end{array}$ & Partition \\
\hline$\rightarrow R$ & $B=-A$ \\
\hline$A \longrightarrow \infty$ & $R=1 / A$ \\
\hline$\rightarrow B$ & $\begin{aligned} R= & R A \\
& (R \cdot \text { Coefficient })\end{aligned}$ \\
\hline$A \longrightarrow P$ & $\begin{array}{l}R=P A \\
\quad(P: \text { Characteristic) })\end{array}$ \\
\hline$\rightarrow R$ & $B=\int A d t$ \\
\hline $\mathcal{J} R$ & $R=\mathrm{d} A / \mathrm{d} t$ \\
\hline & Offset \\
\hline
\end{tabular}

Fig. 1 Main Symbols for Functional Model

\begin{tabular}{|l|l|}
\hline Stiffness & (Velocity) $\rightarrow K$
\end{tabular}$\rightarrow$ (Force)

Fig.2 Main Unit Functional Elements

\section{Single Degree of Freedom Mechanical System}

Figure 3 shows a structural model of single degree of freedom ( $\mathrm{SDOF}$ ) mechanical system ${ }^{(4)}$, where $M$ is mass, $K$ is stiffness, $C_{g}$ is outside viscous damping, $C_{r}$ is inside viscous damping, $v_{1}$ is input velocity, $v_{2}$ is output velocity, $f_{1}$ is output force, $f_{2}$ is input force, $x_{c}$ is spring force and $x_{a}$ is mass velocity. The mathematical model of this SDOF system is as follows.

$$
\left.\begin{array}{l}
M \ddot{x}+C_{g} \dot{x}+C_{r}\left(\dot{x}-\dot{x}_{0}\right)+K\left(x-x_{0}\right)=f_{2} \\
v_{2}=\dot{x} \\
v_{1}=\dot{x}_{0} \\
f_{1}=C_{r}\left(\dot{x}-\dot{x}_{0}\right)+K\left(x-x_{0}\right)
\end{array}\right\}
$$

It is difficult for people who have no preliminary knowledge of dynamics to understand what Fig. 3 means. First, it is not seen what are $M, K, C_{r}$ and $C_{g}$, and what roles they play in the system. Second, it is felt strange and incorrect that displacement of spring, velocity of damper and acceleration of mass are connected directly one another. Third, the direction of force action and the energy flow are

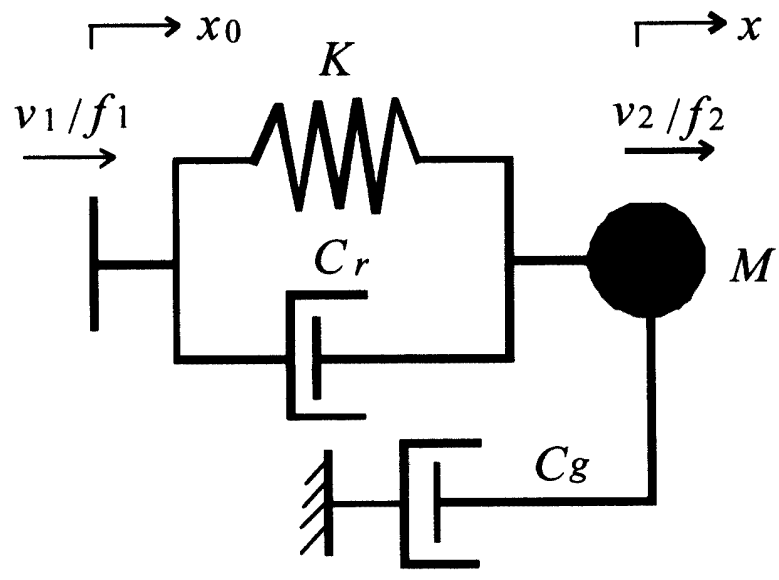

Fig.3 Structural Model of SDOF (Single Degree of Freedom)Mechanical System

not clear. This occurs from the following reason.

The mass yields the force in proportion to the acceleration, the stiffness yields the displacement in proportion to the force, and the damping yields the velocity in proportion to the force. Consequently, these three kinds of characteristics act one another, and as the result, the energy flows in the system. This is the general explanation of the function of the mechanical system. We cannot get this phenomenon from Fig. 3, because Fig. 3 does not explain the function of the SDOF mechanical system. So, the structural model such as in Fig. 3 is not suitable for functional development.

We can accept and draw Fig. 3 smoothly, and can make Eq. 1 referring Fig. 3 without any difficulty. This is because we 
know the above phenomena well in advance as a common sense. It must be almost impossible for people who have no preliminary knowledge about these phenomena to make Eq. 1 referring Fig. 3.

These phenomena can be explained directly and concretely with the functional model proposed by the authors. For example, Fig. 4 is the block diagram with which the functional model of the SDOF system in Fig. 3 is shown.

It can be seen with Fig. 4 clearly and visibly how the characteristics function according to the inputs, the states values, namely the velocity and the force, are generated, the energy flows and the outputs yield.

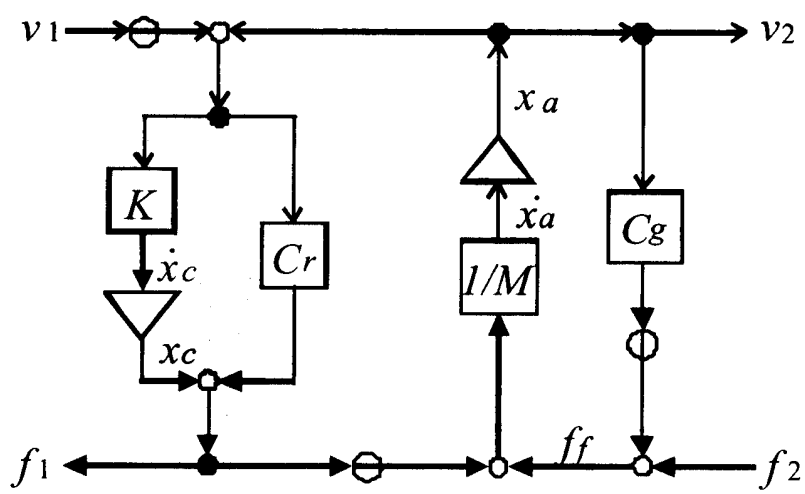

Fig.4 Functional Model of SDOF Mechanical System

The mathematical model is introduced from Fig. 4 as follows, applying the definition of summation in Fig. 1 to the one hollow circle in the upper part of Fig. 4.

$$
\left(\frac{1}{K}\right) \dot{x}_{c}=x_{a}-v_{1}
$$

Concerning the three hollow circles from right to left

$$
\begin{aligned}
& f_{1}=f_{2}-C_{g} x_{a}, M \dot{x}_{a}=f_{1}-f_{1} \\
& f_{1}=x_{c}+C_{r}\left(x_{a}-v_{1}\right)
\end{aligned}
$$

As the self-evident relation

$$
v_{2}=x_{a}
$$

Arranging Eqs. 2 5

$$
\begin{aligned}
& 0=M \dot{x}_{u}+\left(C_{r}+C_{g}\right) x_{u}+x_{r}-C_{r} v_{1}-f_{2} \\
& 0=x_{u}-\frac{1}{K} \dot{x}_{r}-v_{1}
\end{aligned}
$$

Equations 6 and 7 are the equations of force equilibrium and velocity continuity respectively, and these are the state equations. Equations 4 and 5 are the output equations. These four equations are the mathematical model of Fig.4, and equivalent to Eq. 1. Equations $2 \sim 7$ denote that the mathematical model can be obtained with a simple manner using the block diagram of the functional model without any preliminary knowledge on dynamics, differential and integral. Of course, this can be executed automatically with computer.

\section{Single Degree of Freedom Electrical System}

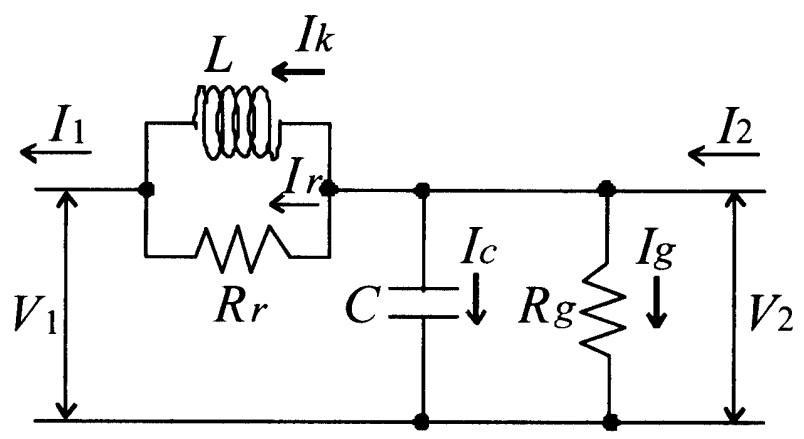

Fig. 5 Structural Model (Circuit Diagram) of SDOF Electrical System

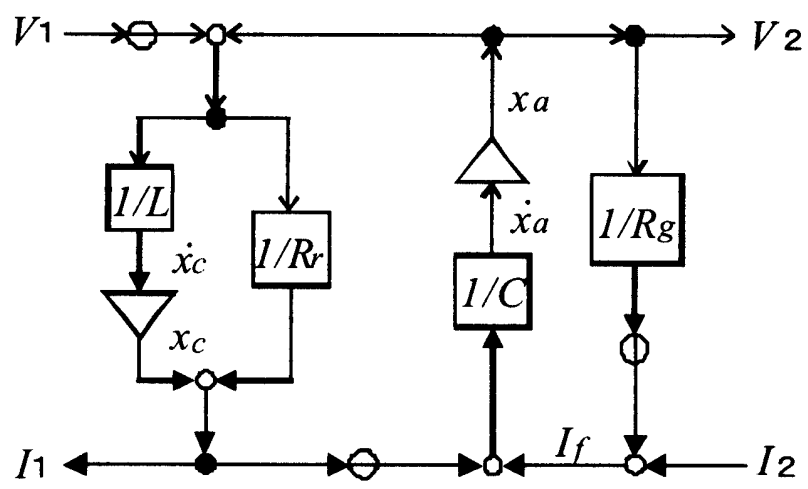

Fig.6 Functional Model of SDOF Electrical System

Figure 5 shows the model of SDOF electrical system with one coil, one condenser and two resistors, in which $V_{1}$ and $I_{2}$ are inputs, $V_{2}$ and $I_{1}$ are outputs. The actual electric circuit can be made connecting these elements according to the structure of the model in Fig. 5, so this is called as the structural model. The mathematical model corresponding to this structural model is 


$$
\begin{aligned}
& I_{2}=I_{r}+I_{g}+I_{k}+I_{r} \\
& I_{r}=C \dot{V}_{2}, \quad V_{2}=R_{g} I_{g} \\
& V_{2}-V_{1}=R_{r} I_{r}=L \dot{I}_{k} \\
& I_{1}=I_{r}+I_{k}
\end{aligned}
$$

It is difficult for people with no preliminary knowledge on electricity to make Eq. 8 referring to Fig. 5, because this figure does not show any function necessary to make Eq. 8.

Figure 6 is the functional model of this electrical system proposed by the authors, and exactly same as that of the mechanical system in Fig. 4, though Fig. 5 is totally different from Fig.3. This means that the functional models become same and common over different engineering specification areas irrespective of physical units, though the structural models become different. So, the mathematical model corresponding to the functional model in Fig. 6 is obtained by the same way as one in Fig. 4 as follows. Applying the definition of summation in Fig. 1 to the one hollow circle in the upper part of Fig. 6

$$
L \dot{x}_{r}=x_{a}-V_{1}
$$

Concerning the three hollow circles from right to left

$$
\begin{aligned}
& I_{1}=I_{2}-\frac{1}{R_{g}} x_{u}, \quad C \dot{x}_{u}=I_{f}-I_{1} \\
& I_{1}=x_{t}+\frac{\left(x_{u}-V_{1}\right)}{R}
\end{aligned}
$$

As the self-evident relation

$$
V_{2}=x_{a}
$$

Arranging Eqs. $9 \sim 12$

$$
\begin{aligned}
& 0=C \dot{x}_{a}+\left(\frac{1}{R_{r}}+\frac{1}{R_{g}}\right) x_{a}+x_{r}-\frac{1}{R_{r}} V_{1}-I_{2} \\
& 0=x_{u}-L \dot{x}_{r}-V_{1}
\end{aligned}
$$

Equations 13 and 14 are the first and the second Kirchhoff's lows, and these are the state equations. Equations 11 and 12 are the output equations. These four equations are the mathematical model of Fig. 6, and equivalent to Eq. 8 .

\section{Wiper System}

Figure 7 shows a wiper system of automobile that is treated

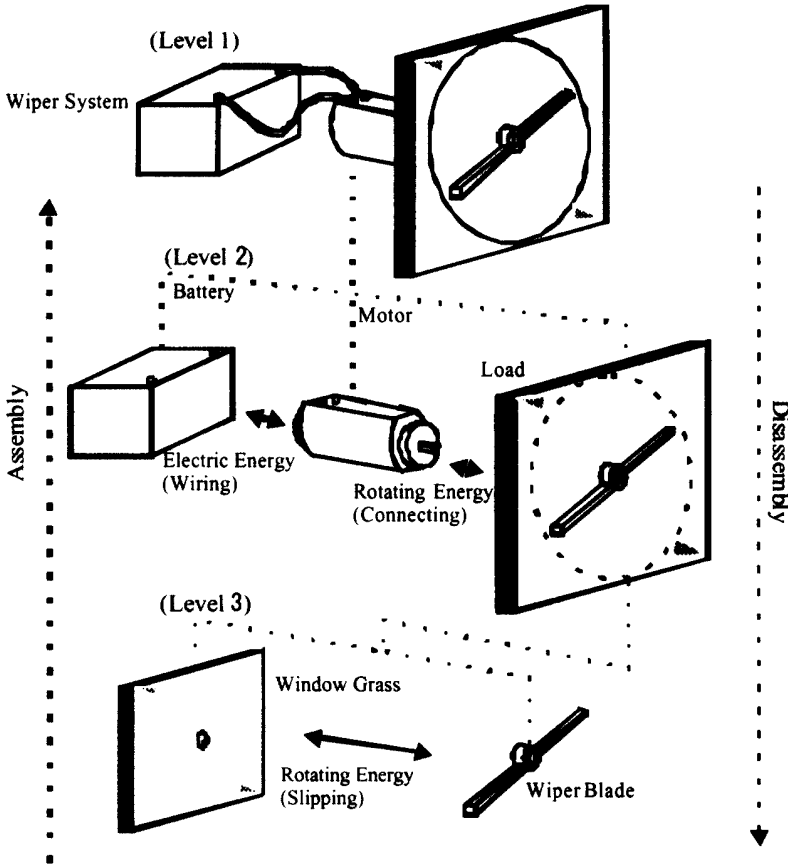

Fig.7 Schematic Figure of Wiper System

as a hierarchical system of three levels ${ }^{(5)}$. The lowest level 3 is composed of two elements, namely wiper blade and window glass. Unifying these two elements, load element in the middle level 2 is made. The middle level 2 is composed of three elements, namely battery, motor and load. Unifying these three elements, the highest level 1 is composed.

Figure 8 shows the functional model of this wiper system. $D_{g}$ is the viscous damping coefficient equivalent to the friction between window glass and blade, $J_{m}$ is the moment of inertia of blade, $D$ is the equivalent viscous damping coefficient of the rotating resistance of motor axis, $J$ is the moment of inertia of rotor, $F$ is the motor constant, $R$ is the electric resistance of motor, $r$ is the inside resistance of battery, $E$ is the inside voltage of battery, $V$ is the terminal voltage of battery, $I$ is the electric current of load, $\omega$ is the rotating angular velocity and $T$ is the rotating torque.

Figure 8 denotes the following strong points of the functional model clearly.

(1) Function of characteristics, relation between voltage and angular velocity, acting direction of electric current, acting direction of torque and energy flow can be got visually. 
(2) Assembling and disassembling of the model can be done in the same sense as to the actual object.

(3) Systems of different physical units such as the electric and the mechanical ones can be connected freely.

(4) Hierarchical model can be composed in correspondence to the subject.

The mathematical model in correspond to Fig. 8 becomes as follows. In level 3

$$
T_{g}=D_{g} \omega_{g}, \quad T_{w^{\prime}}=J_{w} \dot{x}_{w}, \quad \omega_{\mathrm{w}}=\mathrm{x}_{\mathrm{w}}
$$

Unifying Eq. 15, the load in level 2 is

$$
\omega_{l}=\omega_{w}=\omega_{g}, T_{t}=T_{w}+T_{g}
$$

The motor in level 2 is from the three hollow circles in Fig. 8

$$
\left.\begin{array}{l}
J \dot{x}_{m}=F I_{m}-D x_{m}-T_{m} \\
V_{m}=R I_{m}+F x_{m} \quad, \quad \omega_{m}=x_{m}
\end{array}\right\}
$$

The battery in level 2 is

$$
r I_{b}=E-V_{b}
$$

From the condition of energy transfer

$$
\left.\begin{array}{l}
V_{m}=V_{b} \quad, \quad I_{m}=I_{b} \quad, \quad T_{m}=T_{l} \\
\omega_{m}=\omega_{l}=x_{m}=x_{x} \quad, \quad \dot{x}_{m}=\dot{x}_{v^{\prime}}
\end{array}\right\}
$$

Eqs. $15 \sim 19$ can be unified to the SDOF total system of level 1 as follows, eliminating the inside inputs and outputs, $V_{h}$, $I_{m}, \omega_{1}$ and $T_{m}$.

$$
\left[\begin{array}{c}
0 \\
\omega_{m} \\
V_{m} \\
I_{b} \\
T_{l}
\end{array}\right]=\left[\begin{array}{ccc}
-F A E & J+J_{w} & D+D_{g}+F^{2} A \\
0 & 0 & 1 \\
A R E & 0 & F A r \\
A E & 0 & -F A \\
0 & J_{w} & D_{g}
\end{array}\right]\left[\begin{array}{c}
1 \\
\dot{x}_{m} \\
x_{m}
\end{array}\right]
$$

where

$$
A=\frac{1}{r+R}
$$

The highest row of Eq. 20 is the state equation, and the lower four rows are the observing equations.

\section{Conclusions}

A new approach on modeling is presented for functional development of the machine composed of mechanical and electrical systems, and the functional model is proposed to make this new approach possible in the concrete. The properties of model necessary for functional development are listed. The dynamics common to mechanical and electrical systems is introduced. The basic concept of the functional model is explained and illustrated with the mechanical and the electric SDOF systems. An application example of the proposed approach to a wiper system of automobile is presented to verify its validity and usefulness.

\section{References}

1. Sumida S., Nagamatsu M., Hiramatsu S., “Hierarchical Functional Model for Automobile Development", JSAE Review, 19(4), pp.368-371, October 1998.

2. Nagamatsu M., Sumida S., Nagamatsu A., "A New Approach on Modeling for Product Development (The Basic Concept of Functional Model) ", JSME International J., 42(1), pp234-239, March 1999.

3. Nagamatsu A, Sumida S, Nagamatsu M, “A New Approach on Modeling for Product Development (Expansion and Unification) ", JSME International J., 42(4), pp907-913, December 1999.

4. Nagamatsu A, Sumida S, "A New Approach on Modeling for Functional Development of Dynamic System”, Proc. MOVIC'98, 1, pp299-304, August 1998.

5. Nagamatsu A, Sumida S, Nagamatsu M, "A New Approach on Modeling for Virtual Prototype of Automobile", Proc. 32 ${ }^{\text {nd }}$ ISATA, pp83-89, June 1999.

6. Hiramatsu S, Nagamatsu M, Sumida S, “An Approach on Modeling for Functional Development of Automobile" SAE Technical Paper Series 2000-01-0123 (Proc, SAE International Congress 2000), pp.59-71, March 2000.

7. Sumida S, Nagamatsu M, "A Modeling Approach on Modeling of Nonlinear System for Functional Development of Automobile", SAE Technical Paper Series 2001-01-0638 (Proc, SAE International Congress 2001), pp.1-7, March 2001 . 

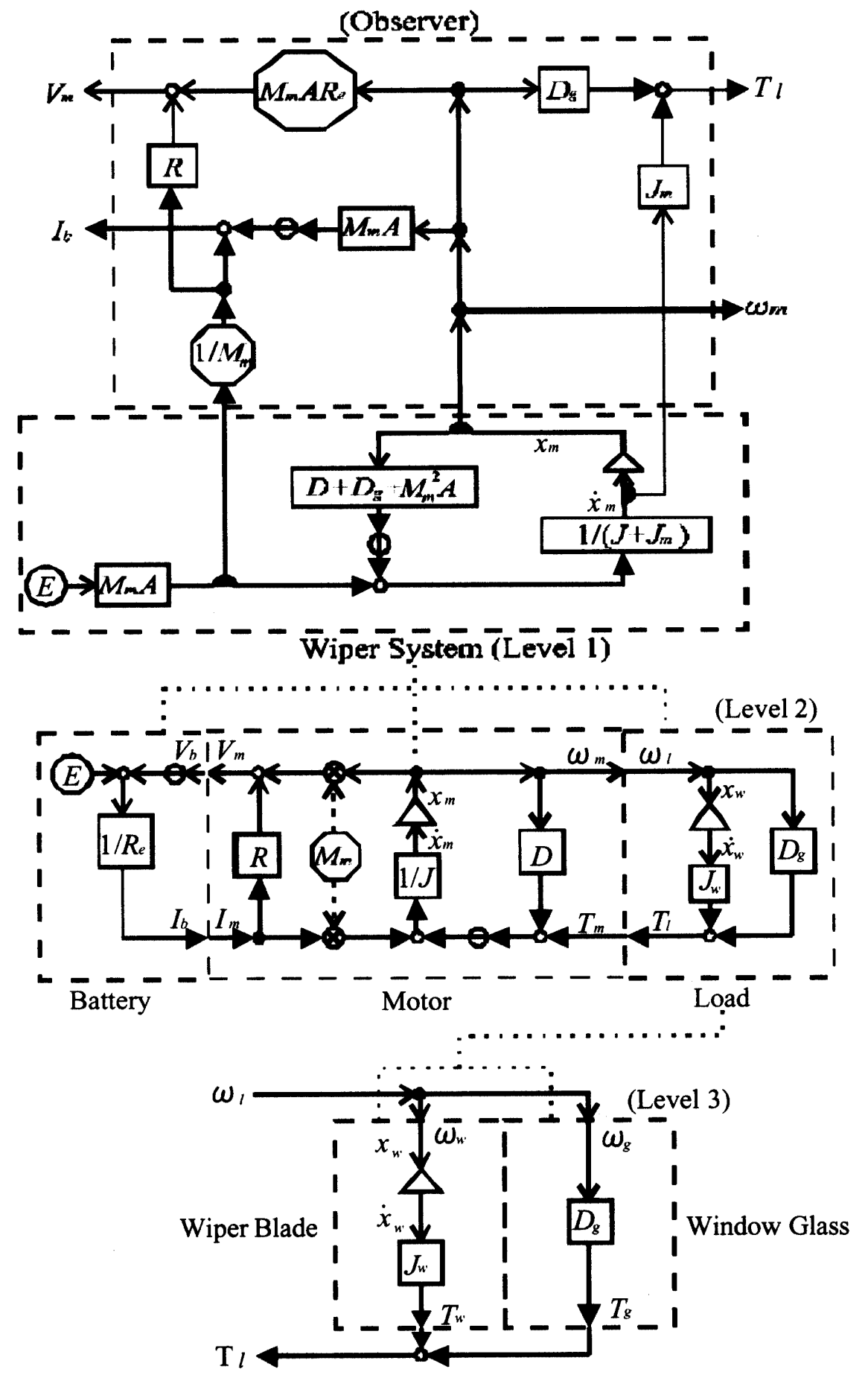

Fig.8 Functional Model of Wiper System 